\title{
From nano-scale to macrostructure in composite for additive technologies
}

\author{
Valentina Anatolyevna Poluektova \\ Department of pure and applied chemistry \\ Belgorod State Technological University named after \\ V.G. Shukhov \\ Belgorod, Russia \\ v.a.poluektova@gmail.com
}

\author{
Nikolay Afanasyevich Shapovalov \\ Department of pure and applied chemistry \\ Belgorod State Technological University named after \\ V.G. Shoukhov \\ Belgorod, Russia \\ val.po@bk.ru
}

\author{
Vladimir Nikolaevich Vladykin \\ Department of unique buildings and structures \\ Belgorod State Technological University named after V.G. Shukhov \\ Belgorod, Russia \\ v.a.poluektova@gmail.com
}

\begin{abstract}
Onrush of additive technologies might allow a 3D concrete printing method to become more profitable and efficient for building unique constructions than traditional methods of building. Until the XXI century, the science paid special attention to phenomena and processes at two levels: microscopic scale (400$760 \mathrm{~nm}$ ) and atomic and subatomic scale (less than $1 \mathrm{~nm}$ ). However, the range from $1 \mathrm{~nm}$ to $100 \mathrm{~nm}$ which is considered within the science of "nano" was not under consideration. But the properties of most materials are defined by their structure at the nanolevel. Considering a mix for 3D printing as a composite material produced from fine aggregate, pastematrix, water and air-bubble voids, it is possible to state that the main problem lies in regulating the process of forming the material structure in a bottom-up way (from nanoscale to macrostructure of the composite material) by nanomodifying the interphase boundary as well as in controlling the kinetics of all chemical reactions accompanying setting processes and optimizing processing activities and service properties of mortars and composites by means of nanomodifiers. It is proved that influencing purposefully the processing properties of high-concentrated mineral suspensions applied in construction industry allows directed synthesis of macromolecules of the nano-size and definite structure which have high absorptive capacity on particle surface and are able to modify the structure of the boundary layer. Modifying cement particles at the nanoscale allows decreasing water requirements of the mix and obtaining the required plastic strength and accelerated strength generation of cement for additive technologies.
\end{abstract}

Keywords - additive manufacturing; three dimensional printing; fine concrete; nanomodifier; phloroglucinol furfural oligomers; nanotechnologies.

\section{INTRODUCTION}

Additive manufacturing or a production technology used to build objects by adding material layer by layer is one of the digital manufacturing areas which develops dynamically. It allows speeding up both $\mathrm{R} \& \mathrm{D}$ and solving preproduction problem, and, in a number of situations, additive manufacturing is actively used for producing single-piece items [1]. In the world practice, the term "additive manufacturing" refers to processes where $3 \mathrm{D}$ printing is applied [2].

Most processes of 3D printing are energy-consuming and they use petroleum-based plastic material which is not subject to recycling. Until recently, thermoplastic polymers were mainly used as printing "ink" for 3D printers. However, using thermoplastic materials for manufacturing large-scale items is unprofitable; therefore, scientists have focused on applying materials which are available in a fine powder form [3, 4].

Specialists of MarketsandMarkets company presented a report which stated that the global market for 3D concrete printing would grow two times in five years reaching $\$ 56.4$ millions. It is connected with the fact that additive manufacturing technologies are rapidly developed and 3D concrete printing may appear to be more profitable and efficient for building unique constructions than traditional methods of building (Fig. 1) [4, 5]. 


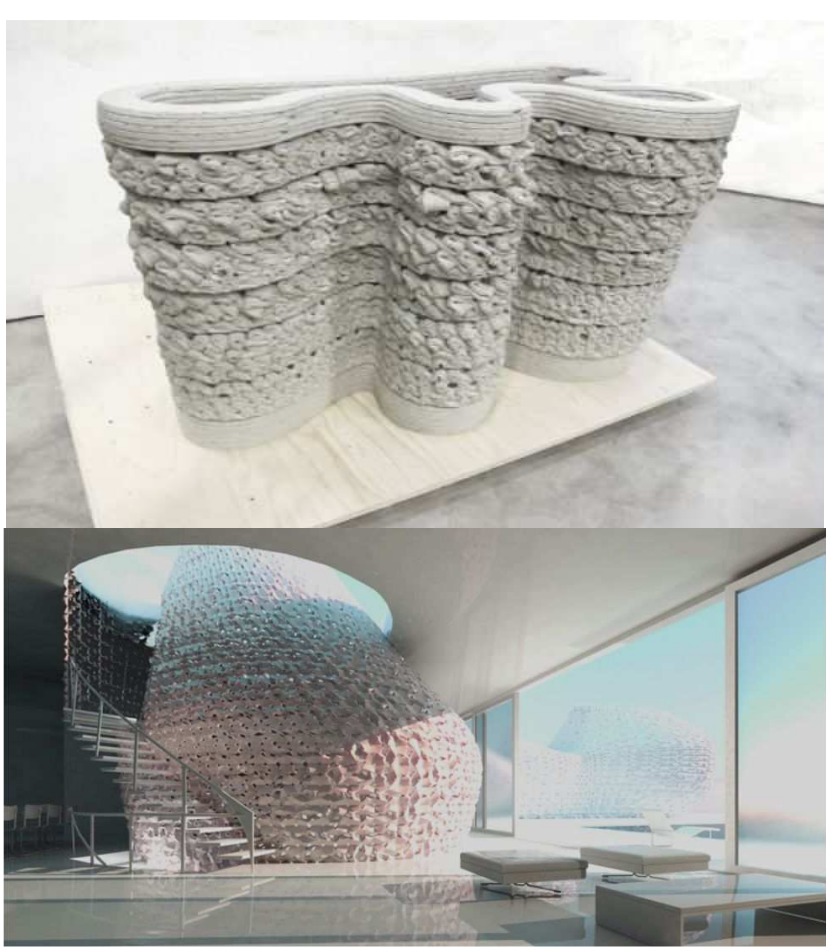

Fig. 1. An example of creative 3D printing application in construction industry [4]

Concrete mixes used in modern construction industry with the help of additive technologies require special processing properties: high plastic strength, accelerated curing and the ability to increase initial concrete strength. These problems could be solved by optimizing concrete composite properties by means of controlling the processes of its structure formation at the nanolevel.

Until the XXI century, the science paid special attention to phenomena and processes at two levels. The first level is microscopic scale (everything that can be seen, i.e. more than optical wavelength $400-760 \mathrm{~nm}$ ). The second level is atomic and subatomic scale (less than $1 \mathrm{~nm}$ ). In this connection, the range from $1 \mathrm{~nm}$ to $100 \mathrm{~nm}$ which is considered within the science of "nano" was not under consideration. However, properties of most materials except for single crystals (atomic range, i.e. sub-nanometer scale) are defined by their structure at the nanolevel. The applicability of traditional technologies based on the top-down approach reached its limitations, thus the idea to apply the bottom-up approach arose which meant to use molecular self-organization at the nanoscale.

Nanotechnology is a new scientific area which is at its initial stage of development: information and knowledge are used only in labolatories. Nevertheless, nanotechnology is developing rapidly [6-8].

Considering a mix for $3 \mathrm{D}$ printing as a composite material produced from fine aggregate, pastematrix, water and airbubble voids, it is possible to state that the main problem lies in regulating the process of forming the material structure in a bottom-up way (from nanoscale to macrostructure of the composite material) by nanomodifying the interphase boundary as well as in controlling the kinetics of all chemical reactions accompanying setting processes and optimizing processing activities and service properties of mortars and composites by means of nanomodifiers.

Using materials which contain nanoparticles in its composition gives an obvious advantage relating not only to improvements in the properties of usual products applied in everyday life but also to creating new ones which are entirely different from those produced earlier by man. In this way, the research conducted in some European research institutions excites interest. The researchers suggest adding myriads of polymeric nanoparticles into concrete mix developed in such a way that when being under pressure they can turn into liquid which is able to penetrate into cracks, and then to strengthen, like glue or concrete, thereby enhancing seismic stability of constructions by stabilizing load-bearing structures [9].

Belgorod State Technological University named after V.G. Shukhov (BSTU) have positive experience in getting nanoparticles based on trifunctional oxyphenol. Adding synthesized oligomers into concrete mix at the initial stage allows controlling the kinetics of interaction between cement and mixing water and achieving maximum positive effects at the following phases:

- regulating rheological properties and aggregative stability of suspensions;

- colloiding, providing the required persistence of fluidity in time;

- crystallizing, by means of enhancing heterophase boundaries of contact zones and thereby increasing concrete strength.

Purposefully influencing on processing properties of highconcentrated mineral suspensions applied in construction industry allows directed synthesis of macromolecules of nano size and definite structure which have high absorptive capacity on particle surface and are able to modify the structure of the boundary layer $[8,10]$. Absorptive capacity of organic compounds depends, in the first instance, on the hydrocarbon chain length and molecular weight of a compound.

\section{METHODOLOGY}

Nanomodifier synthesis was made in a three-necked reaction flask equipped with a stirrer, a thermometer and a reflux condenser.

To determine the composition and the structure of the oligomeric molecules of the nanomodifier, ultraviolet spectroscopy, infrared spectroscopy, nuclear magnetic resonance spectroscopy and conductometry have been applied.

A conductometric back titration method was used to determine the quantity of phenolic hydroxyl groups in the molecule. The measurement of phenolic oxy groups was carried out according to the following formula: 


$$
n_{\text {phen }}=\frac{\left(V_{2}-V_{1}\right) \cdot N \cdot M}{C_{o l} \cdot V_{o l}}
$$

where $n_{\text {phen }}$ - the number of phenolic OH-groups in the oligomer molecule; $V_{1}$ - the volume of $\mathrm{HCl}$ used to titrate the excess of alkali; $V_{2}$ - the total volume of $\mathrm{HC} 1$ used to titrate the alkaline solution of the oligomer; $N$ - the normality of $\mathrm{HC1} ; M$ - the molecular weight of the oligomer; $C_{\mathrm{ol}}-$ oligomers concentration, $\mathrm{kg} / \mathrm{m}^{3} ; V_{\mathrm{ol}}$ - the volume of oligomer solution.

The molecular weight of the synthesized oligomers has been determined by applying a cryoscopic method.

Oligomers adsorption on particulates has been studied by applying ultraviolet spectrometer SPECORD UV in the ultraviolet area where $v=50 \cdot 10^{3} \mathrm{sm}^{-1}$ according to the decrease of concentrations of the test oligomers in the dispersion medium after adsorption equilibrium was set.

The area $S_{0}$ per one molecule in the saturated adsorption layer was calculated by using the following equation:

$$
\begin{aligned}
& S_{0}=\frac{1}{N_{A} G_{\infty}} \\
& \alpha+\beta=\chi
\end{aligned}
$$

where $G_{\infty}-$ maximal adsorption, $\mathrm{kg} / \mathrm{m}^{2} ; N_{A}-$ Avogadro number.

The thickness of adsorption layers $\delta$ was calculated by using the following formula:

$$
\begin{gathered}
\delta=\frac{G_{\infty}}{\rho} \\
\alpha+\beta=\chi
\end{gathered}
$$

where $\delta-$ the thickness of the adsorption layer, m; $\rho-$ the density of adsorbate, $\mathrm{kg} / \mathrm{m}^{3}$.

The object of nanomodification research was samples of fine concrete. To prepare the sand-cement samples, the following materials have been used: cement - PC-500-DO by Belgorod cement plant with specific surface $354 \mathrm{~m}^{2} / \mathrm{kg}$, sand from Belgorod sandpit with fineness modulus 1.2, moisture $2.3 \%$, density $2600 \mathrm{~kg} / \mathrm{m}^{3}$.

To research the influence of the nanomodifier on the properties of mortar mixes and fine concretes, multimeter methods of testing regulated by the corresponding state standards specifications have been applied. The concentration of the modifier was calculated in mass \% according to dry unit weight depending on a number of dispersed phases.

\section{BASIC PART}

While doing this research, the thermally-reactive oligomer based on trifunctional phenol (phloroglucinol) was synthesized. Furfural was used as a condensing agent. The synthesis was conducted in presence of alkaline catalysts to get thermally-reactive oligomers.

\section{A. Obtaining a phloroglucinol furfural nanomodifier}

The scheme of synthesis by means of polycondensation of phloroglucinol and furfural in an alkaline medium can be presented in the following way:

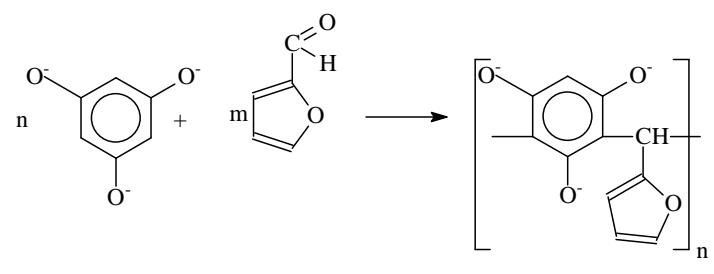

As a result of reactions, thermally-reactive oligomers of different apparent molecular weight were obtained. Their composition and structure were determined by the correlation of the initial reagents, their concentrations and temperature. Changes in temperature influenced the reaction rates considerably.

Simultaneous determination of plasticization ability of the reaction products revealed that in the beginning the molecular weight and the plasticization ability increase symbiotically, but starting from the molecular weight 900-950, its further increase does not contribute to plasticization ability growth. It indicates that increasing chain length of oligomer molecules influences the plasticization ability only until a certain level.

The analysis of results allowed defining the optimal relationship of reagents in order to get a phloroglucinol furfural modifier with the most plasticization ability.

Studying the composition and structure of the modifier by means of ultraviolet, infrared, nuclear magnetic resonance spectra revealed that in oligomer spectra, there is a decrease of proton quantity in the aromatic ring, but $\mathrm{OH}$-groups remain.

The conductometric back titration method was used to determine a number of phenolic hydroxyl groups in a molecule. The calculation showed that a number of $\mathrm{OH}$ groups per one molecule are $18 \pm 0.5$; besides, its number remains constant in a structural repeating unit of the macromolecule in comparison with phloroglucinol. It is a direct proof that phenolic OH-groups do not take part in polycondensation reaction. In that way, the molecular structure of the phloroglucinol furfural modifier can be represented as a following model (Fig. 2).

It is known that all $\mathrm{C}-\mathrm{C}$ bonds in the benzene ring are equal, their length is $0.140 \mathrm{~nm}$ that corresponds to the intermediate value between the length of a single linkage $(0.154 \mathrm{~nm})$ and the length of a double one $(0.134 \mathrm{~nm})$. On the basis of these facts, the size of the benzene molecule is assumed to be about $0.5 \mathrm{~nm}$. The $\mathrm{C}-\mathrm{O}$ bond length is equal to $0.126 \mathrm{~nm}$. The atomic radii of carbon and oxygen are equal to 0.077 and $0.066 \mathrm{~nm}$ respectively. Using the values of atomic radii or bond lengths obtained by summing atomic radii, the length $L$ of the expected unfolded molecule of the modifier was calculated as equal to $6.8 \mathrm{~nm}$. 


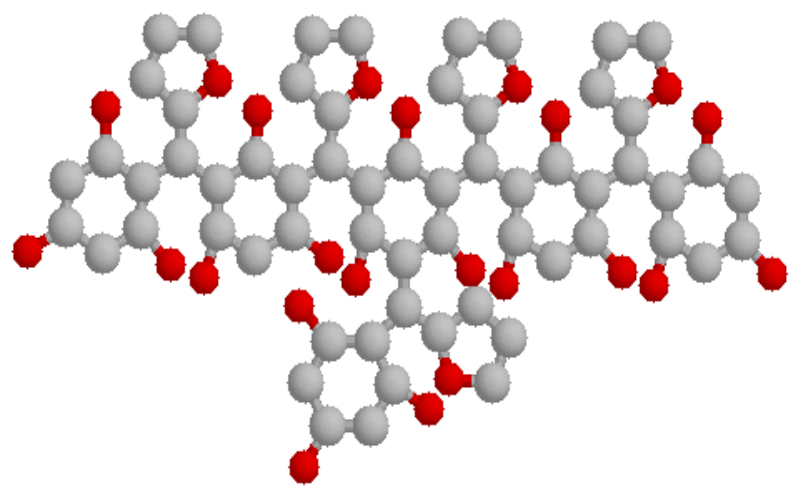

Fig. 2. The model of the molecular structure of the phloroglucinol furfural modifier

Thus, on the basis of phloroglucinol furfural oligomers, the modifier whose molecules are of nano size was obtained.

\section{B. Adsorption of nanomolecules of phloroglucinol furfural modifier}

While researching, the adsorption isotherms of the phloroglucinol furfural oligomers on the surface of mineral particles which have typical characteristics of monomolecular adsorption were obtained.

The conducted research allowed figuring on some adsorption parameters: calculation of the surface area occupied by one molecule $\left(2.54 \mathrm{~nm}^{2}\right)$ and calculation of adsorption layer thickness of phloroglucinol furfural oligomers $(0.67 \mathrm{~nm})$.

The obtained values of adsorption parameters and correlation with the design value of the linear length of the molecules of the synthetized oxyphenol oligomers confirm that gradual increase of oligomers length contributed to the appearance of a new quality peculiar to polymers - flexibility of macromolecules.

Figure 3 shows a 3D model of the computed conformation of the synthesized molecule with the help of SymApps software by Bio-Rad Laboratories company.

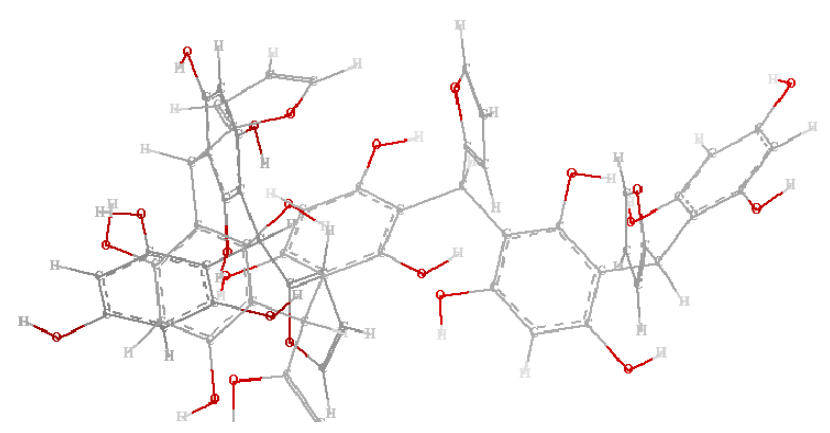

Fig. 3. A computed 3D molecular structure of the phloroglucinol furfural nanomodifier

It is known that the rotation of particular atomic groups around valence bond directions in molecules of even small length results in appearance of a big number of particular stereoisomers, the so-called rotational isomers (rotamers). The rotation of particular units of the molecule happening under the influence of thermal motion is carried out without significant changes of valence angles and interatomic distances. The variations in their values are within $2-3 \%$.

While adsorbing the nanomodifier on the surface of mineral particles one could observe surface hydrophilization. Consequently, the molecules are adsorbed in such way that one part of anion-active groups interacts with the particle surface while the other part of hydrophilic anion-active groups faces the solution.

Studying the process of adsorbing molecules of the synthesized nanomodifier on the surface of the particles of the cement-sand grout revealed that adsorption is supported by ionic interaction of negative oxy groups of phloroglucinol link with positively charged active surface centres of cement and sand and by dispersion force of interaction between aromatic ring system of oligomer and the surface of particles. At the same time, the peptization of aggregates of mineral particles up to their initial sizes with release of immobilized water can be observed.

In that way, the molecules of the nanomodifier adsorbed on the surface of the particles of dispersed materials, forming the monomolecular layer, modify the interphase boundary of mineral suspensions at the nanolevel.

\section{Macrostructure and properties of a nanomodified composite}

When designing a mortar formula for 3D printing, i.e. for producing items without concrete form by applying additive technologies, it is important to study the influence of the synthesized nanomodifier on plastic strength of the cement paste and time of set. Figure 4 represents the comparison characteristics of plastic strength of the reference and modified mortar mixes.

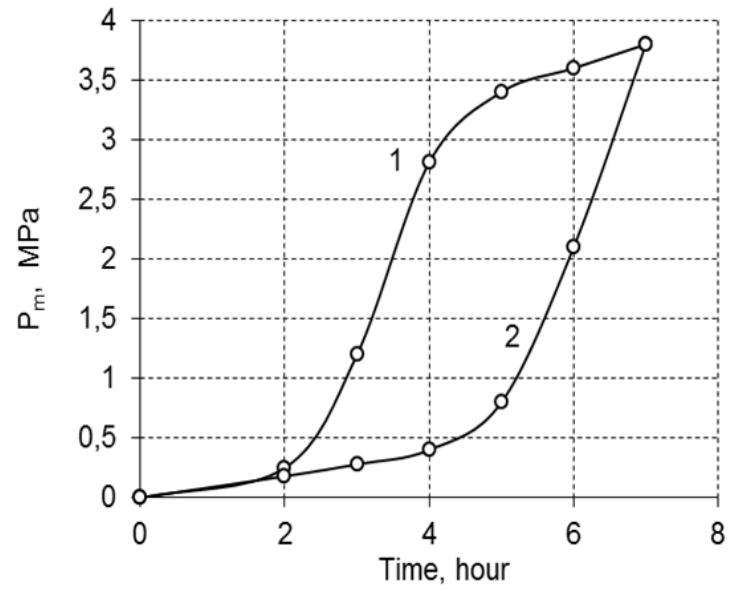

Fig. 4. The change of plastic strength of the cement paste with time when the water-to-cement ratio is fixed: 1 - reference mortar mix; 2 - modified mortar $\operatorname{mix}$

The modified mortar mixes demonstrate some increase in starting date of strength generation during the first 6-8 hours. 
This is connected with the increase of water-retention value of the modified surface of the cementing due to the oligomers molecular adsorption and contact surface expansion as a result of aggregates peptization. At a later stage, hardening of the modified cements intensifies in comparison with the reference mix. The increase in starting date of strength generation is unacceptable for additive manufacturing. However, the synthesized nanomodifier possesses super water reducing properties. The research showed that the synthesized nanomodifier based on phloroglucinol furfural oligomers up to State Standard 24211-2008 can be rated as a superplasticizer and high range water reducer. In respect of its superplasticizing and water reducing capacities, the synthesized nanomodifier is as good as well-known domestic and foreign analogs.

The results of studying the properties of the nanomodified composite with the reduced content of mixing water are represented in Table 1.

TABLE I. PROPERTIES OF FINE AGGREGATE MIXTURES AND CONCRETES

\begin{tabular}{|c|c|c|c|}
\hline $\begin{array}{c}\text { Modifier } \\
\text { percentage, \% }\end{array}$ & $\begin{array}{c}\text { Water-to-cement } \\
\text { ratio }\end{array}$ & $\begin{array}{c}\text { Slump, } \\
\text { cm }\end{array}$ & $\begin{array}{c}\text { Ultimate } \\
\text { compression } \\
\text { strength, MPa } \\
\text { (28 days) }\end{array}$ \\
\hline- & 0.45 & 3 & 39.5 \\
\hline 0,20 & 0.34 & 3 & 60.2 \\
0,20 & 0.45 & 20 & 30.1 \\
\hline
\end{tabular}

Ttable 1 shows that it is possible to reduce water requirement for modified mortar mixes by $25 \%$ compared with plain cement. Water requirements could be reduced more by increasing the modifier content added with mixing water. However, the selection criterion of optimal conditions was within $0.33 \ldots 0.35$ in order to obtain mortar mixes of the required plasticity for additive manufacturing, since further reduction of water-to-cement ratio can reduce concrete durability. [11].

The kinetics of strength generation of mixes with equal fluidity is shown in Table 2 .

According to the given information, initial hardening retardation of concrete is not typical for the modified concrete mix. At the same time, hardening of the modified cements intensifies in 1-2 days in comparison with the reference mix.

TABLE II. THE DEPENDENCE OF CONCCRETE STRENGTH ON TIME OF HARDENING

\begin{tabular}{|c|c|c|c|c|c|c|c|c|}
\hline \multirow{3}{*}{ 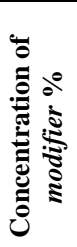 } & \multirow{3}{*}{ 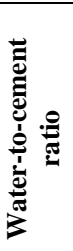 } & \multirow{3}{*}{ 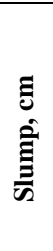 } & \multicolumn{6}{|c|}{ Ultimate working capacity, MPa } \\
\hline & & & \multicolumn{3}{|c|}{ Compression strength } & \multicolumn{3}{|c|}{ Flexural strength } \\
\hline & & & 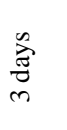 & $\sum_{n}^{\infty}$ & 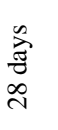 & $\begin{array}{l}\stackrel{\infty}{\vec{z}} \\
m\end{array}$ & $\sum_{\pi}^{\infty}$ & 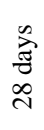 \\
\hline- & 0.45 & 3.0 & 18.5 & 25.3 & 39.5 & 1.9 & 3.0 & 5.5 \\
\hline 0.2 & 0.34 & 3.0 & 31.1 & 41.6 & 60.2 & 2.2 & 4.1 & 7.5 \\
\hline
\end{tabular}

The accelerated strength generation of cement of the given mix at an initial stage can contribute positively to the behavior of innovative materials and final products obtained with the help of additive technologies.

The results of studying the stone microstructure from the modified mortar mixes showed that more compact fine crystalline structure without large-block connections and visible defects is formed. This can be explained by peptizating effect of the nanomodifier and by obtaining more fine crystalline structure of the concrete. In addition, the concrete strength is higher compared with the reference samples. Thus, the modified concretes in comparison with the reference sample without a modifier have a strength increase of more than $20 \mathrm{MPa}$.

\section{CONCLUSION}

Studying theoretical and practical characteristics of phloroglucinol furfural oligomers synthesis, defining optimal synthesis conditions, determining the composition and structure by methods of infrared, nuclear magnetic resonance spectroscopy, conductometry, calculating the length of the unfolded oligomer molecule allowed obtaining compounds which can be rated as nanomodifiers of mineral suspensions.

The conducted research revealed that the characteristics of the boundary nanolayer structure are defined by adsorbing oligomers on the solid surface. It allows influencing purposefully on the aggregate size of mineral particles and plasticization of construction suspensions.

The nanomodifier based on phloroglucinol furfural oligomers possesses superplasticizing and water reducing capacities, accelerates strength generation at the early stage of hardening, and improves concrete strength rates.

\section{FINDINGS}

The results of the given paper show that the nanomodifier can be used when producing high-strength innovative materials for additive manufacturing. Modifying cement particles by the super plasticizing agent at the nanoscale allowed decreasing water requirements of the mix by $25 \%$ and getting the required plastic strength for additive technologies.

The modified concrete demonstrates more intensive development of strength in 1-2 days of hardening in comparison with the reference mix. Besides, the more compact fine crystalline structure without large-block connections and visible defects is formed. In addition, modifying concretes at the nanolevel allows a strength increase of more than $20 \mathrm{MPa}$.

The obtained results can be applied when creating innovative mixes for 3D printing.

\section{Acknowledgment}

The work is realized in the framework of Russian Federal Target Program № 14.577.21.0193 of 28.10.2015; the Program of flagship university development on the base of the Belgorod 
State Technological University named after V.G. Shoukhov, using equipment of High Technology Center at BSTU named after V.G. Shoukhov.

\section{References}

[1] K. Kazmirchuk, V. Dovbysh, "Additive Technologies in Russian Industry" , 2012 [Electronoc resource: http://konstruktor.net/podrobneedet/additivnye-texnologii-v-rossijskoj-promyshlennosti.html].

[2] M.A. Zlenko, A.A. Popovich, I.N. Mutylina, "Additive Technologies in Machine Building. Teaching Aid," SPb.: SPbSU, 2013, p. 221.

[3] A. Maslov, "3D printing in Construction. Mixes. Practical guidelines," 2016 [Electronoc resource: http://specavia.pro/articls/Stroitelnaja-3Dpechat-Smesi-Prakticheskie-rekomendacii/[.

[4] Market Research Reports: Date Views 01.02.2016 [Electronoc resource: http://www.marketsandmarkets.com/[.
[5] N. Vatin, L. Chumadova, I. Goncharov, V. Zykova, A. Karpenya, A. Kim, E. Finashenkov, "3D printing in construction," Construction of Unique Buildings and Structures, 1(52), pp. 27-46, 2017.

[6] S.Yu. Glazyev, V.V. Kharitonov, Nanotechnologies as a key factor of a new technological structure in economics, Moscow, Trovant, 2009.

[7] A.N. Ponamaryev, "Quality concretes. Possibility analysis and practice of applying nanotechnological methods," Construction engineering journal, №6, pp. 25-33, 2009.

[8] N.A. Shapovalov, V.A. Poluektova, "Some aspects of nanomodification of mineral dispersions by oligomers based on trifunctional oxyphenyl," Nanotechnologies in Construction, 8(6), pp. 43-57, 2016.

[9] "Concrete of a new age will beautify the millennium", 2009, Vlastra, 7(93), pp.8-9

[10] N.A. Shapovalov, V.A. Poluektova, "Features of nanomodifiers synthesis based on trifunctional oxyphenyls for mineral suspensions," Nanotechnologies in Construction, 8(5), pp. 100-115, 2016.

[11] V.V. Babkov, A.F. Polak, P.G. Komokhov, "Aspects of pastematrix durability," Cement, 3, pp. 14-16, 1988. 